\title{
Functional analysis of the GRMZM2G174449 promoter to identify Rhizoctonia solani-inducible cis-elements in maize
}

\author{
Fangfang Yang ${ }^{1 \dagger}$, Xinhua Ding ${ }^{1,2+}$, Jing Chen ${ }^{2}$, Yanting Shen ${ }^{1}$, Lingguang Kong ${ }^{2}$, Ning Li ${ }^{1 *}$ and Zhaohui Chu ${ }^{1 *}$ (D)
}

\begin{abstract}
Background: Banded leaf and sheath blight (BLSB), caused by the necrotrophic fungus Rhizoctonia solani, is a highly devastating disease in most maize and rice growing areas of the world. However, the molecular mechanisms of perceiving pathogen signals are poorly understood in hosts.

Results: Here, we identified a Rhizoctonia solani-inducible promoter pGRMZM2G174449 in maize. Deletion analysis showed that the -574 to -455 fragment was necessary for $p G R M Z M 2 G 174449$ in responding to $R$. solani and this fragment contained the unknown pathogen-inducible cis-elements according to the bioinformatics analysis. Furthermore, detailed quantitative assays showed that two cis-elements, GCTGA in the -567 to -563 region and TATAT in the -485 to -481 region, were specifically responsible for the $R$. solani-inducible activity. A series of point mutation analysis indicated that the two cis-elements have the conserved motifs of NHWGN and DWYWT, respectively.

Conclusion: Our results indicated that $P G R M Z M 2 G 174449$ is a good $R$. solani-inducible promoter suitable for genetic engineering of BLSB resistance. And NHWGN and DWYWT are two $R$. solani-inducible cis-elements that play important roles in $P G R M Z M 2 G 174449$ responding to $R$. solani.
\end{abstract}

Keywords: Maize, Banded leaf and sheath blight, Rhizoctonia solani, cis-element, Genetic engineering

\section{Background}

Pathogen attacks have drastic effects on crop growth and development, which significantly limit agricultural productivity. To enhance crop disease resistance, cloning of key genes related to pathogen invasion and precisely regulating transgene expression are important measures for solving the problem [1]. In current applications, constitutive promoters, such as the cauliflower mosaic virus 35S (CaMV35S) and ubiquitin promoters, have been frequently used to assess the effects of transgene expression in many plant species. However, in certain cases, the constitutive overexpression of defense-related genes may result in negative effects on crop growth and yields [2-4]. Therefore, cloning and identifying characterizing pathogen-inducible

\footnotetext{
*Correspondence: nli1986@163.com; zchu@sdau.edu.cn

${ }^{\dagger}$ Equal contributors

${ }^{1}$ State Key Laboratory of Crop Biology, College of Agronomy, Shandong Agricultural University, Tai an 271018, Shandong Province, People's Republic of China

Full list of author information is available at the end of the article
}

promoters are the keys to understanding the regulation mechanisms of defense-related genes, and these promoters could be the most useful types of promoters for engineering crop lines with enhanced and durable disease resistance [5].

Many pathogen-inducible promoters have been identified [6-8], and some of them have been used to generate high-quality transgenic plants [9-11]. Pathogen-inducible promoters usually possess many conserved cis-elements that are potential binding sites for pathogen-responsive transcription factors. Among them, the GCC-like elements $[12,13]$ and the W-box [14-16] are two groups of cis-regulatory elements that have been widely studied and functionally validated. Beside the GCC-like elements and W-box, some other pathogen-inducible cis-elements have also been identified, such as the S-box [17], G-box [18], E-box [19], PRE2 and PRE4 [20] as well as the MYB recognition elements [8]. Some of these elements, such as the Wbox, GCC-box and S-box, have been used to construct synthetic pathogen-inducible promoters $[9,21]$. 
Banded leaf and sheath blight (BLSB), which is caused by the necrotrophic fungal pathogen Rhizoctonia solani, often leads to extensive necrosis in the leaf sheaths of hosts, eventually causing the death of the infected plant and resulting in substantial economic losses. It is a typical soil-born and annual cumulative disease which widely spread out in most maize and rice growing areas of the world $[22,23]$. To date, sheath blight resistance has been demonstrated to be controlled by minor-effect QTLs [24, 25], and only a few defense-related genes have been identified [11, 26-29]. Recent study showed that OsASR2 could regulate the response of Os $2 \mathrm{H} 16$ gene to $R$. solani by targeting the GT-1 cis-element [30]. However, understanding of the regulation mechanisms of $R$. solani-inducible genes is still very limited, with a few $R$. solani-inducible promoters and three cis-elements have been reported recently [30-34].

In this study, we focused on a maize $R$. solani-induced expression gene, GRMZM2G174449. To better understand how the GRMZM2G174449 gene is regulated, we completely analyzed the promoter of GRMZM2G174449 by using $\beta$-glucuronidase (GUS) or green fluorescent protein $(G F P)$ gene as reporter genes. We found that the 5 '-flanking sequence of the GRMZM2G174449 promoter could be induced by $R$. solani inoculation. Deletion analysis showed that two novel cis-elements, GCTGA and TATAT, were specifically responsive to $R$. solani infection. Mutation analysis indicated that these two elements have the conserved motifs of NHWGN and DWYWT, respectively. These results will improve our understanding of GRMZM2G174449 regulation and increase the number of promoter and cis-elements available for potential use in development of transgenic plants with enhanced $R$. solani resistance.

\section{Methods}

Plant materials and pathogens

Rice cultivar Zhonghua 11 and maize inbred line B73 were grown in greenhouse at $28{ }^{\circ} \mathrm{C}$ with a $16 / 8 \mathrm{~h} \mathrm{light/}$ dark cycle. Nicotiana benthamiana (NB) was grown in chamber at $25{ }^{\circ} \mathrm{C}$ under a $16 / 8 \mathrm{~h}$ light/dark cycle. For the tissue expression analysis of GRMZM2G174449 and its promoter assay, tissues were harvested for total RNA isolation and GUS assay, including with young root, young culm, young leaf, root, culm, leaf, anther, pistil and endosperm tissues. As previously reported [34], $R$. solani strains were grown in Potato-DextroseBroth media (potato at $200 \mathrm{~g} / \mathrm{L}$, glucose at $20 \mathrm{~g} / \mathrm{L}$ and agar at $15 \mathrm{~g} / \mathrm{L})$ at $25^{\circ} \mathrm{C}$ for 3 days. Magnaporthe grisea strain RB21 was grown in Rice Bran media (rice bran at $20 \mathrm{~g} / \mathrm{L}$, yeast powder at $2 \mathrm{~g} / \mathrm{L}$ and agar at $15 \mathrm{~g} / \mathrm{L}$ ) at $25{ }^{\circ} \mathrm{C}$ for 10 days. Xanthomonas oryzae pv. oryzae strain PXO99 and Xanthomonas oryzae pv. oryzicola strain RS105 were grown in Polypeptone-Sucrose-Agar media (polypeptone at $10 \mathrm{~g} / \mathrm{L}$, glutamic acid at $1 \mathrm{~g} / \mathrm{L}$, sucrose at $10 \mathrm{~g} / \mathrm{L}$ and agar at $15 \mathrm{~g} / \mathrm{L}$ ) at $28{ }^{\circ} \mathrm{C}$ for 2 days and then suspended in sterile water to $\mathrm{OD}_{600}=0.5$. Infected and non-infected leaves were harvested for the GUS and GFP assays.

\section{Vector constructions of the GRMZM2G174449 promoter and its deletion derivatives}

The full-length GRMZM2G174449 promoter was amplified from the maize inbred line B73 based on the maize genome sequence (http://www.maizegdb.org/ gbrowse/maize_v4) with the primers listed in Additional file 1: Table S1. To generate the GRMZM2G174449 promoter assay construct, the appropriate restriction sites were introduced into the PCR-amplified promoter ( $\mathrm{Sal}$ I at the $5^{\prime}$ end; BamH I at the 3 ' end), and then cloned into double digested pCAMBIA1391-Sal I-BamH I-cut, which was named pC1391 D0. The deleted promoters were cloned into the $p C X G U S-P$ and $p C X G F P-P$ vectors as previously described $[30,35]$ to generate deletion constructs containing various fragments $(-1518$ to +46 , pCXGUS D1; -1114 to +46 , pCXGUS D2; -694 to +46 , pCXGUS D3; -454 to +46 , pCXGUS D4; -274 to +46 , pCXGUS D5; -574 to +46 , pCXGUS D6; -574 to -455 , pCXGFP delA; -574 to -550 , pCXGFP delB; -549 to -528 , pCXGFP delC; -527 to -491 , pCXGFP delD; -490 to -478 , pCXGFP delE; -477 to -455 , pCXGFP delF). To identify the GCTGA and TATAT cis-elements, the sequence of two elements were repeated twice, fused with the $35 \mathrm{~S}$ minimum promoter and named $2 \times$ GCTGA and $2 \times$ TATAT, respectively. For mutation analysis, the mutations of GCTGA and TATAT were repeated twice and fused with the $35 \mathrm{~S}$ minimum promoter as described above. All the primers were listed in Additional file 1: Table S1.

\section{Rice transformation}

For promoter analysis, the pC1391 D0, $2 \times$ GCTGA and $2 \times$ TATAT constructs were transformed into rice cultivar Zhonghua 11 to generate the transgenic plants. Agrobacterium tumefaciens-mediated method was used for rice transformation with mature embryos and $A$. tumefaciens strain EHA105 [36]. Positive selection and validation were performed with PCR basing on GUS or GFP genes, and three $\mathrm{T}_{1}$ lines of each were used for further analysis.

\section{Transient expression in Nicotiana benthamiana and quantification of GUS and GFP}

Transient expression in NB leaves was performed according to a previously described method [30, 34]. Histochemical GUS staining of transgenic rice leaves was performed as described previously [37]. The leaves 

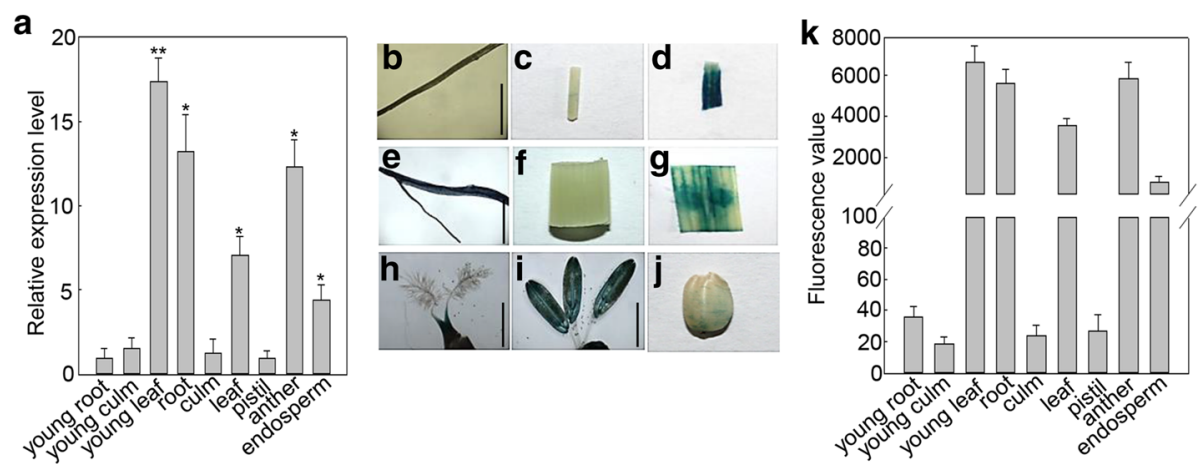

Fig. 1 Tissue expression analysis of the maize gene GRMZM2G174449 and its promoter. a Tissue-specific analysis of GRMZM2G174449 in maize. The significant differences were compared with young root. Asterisks indicate statistically significant differences, as determined by Student's t-tests $\left({ }^{*} P<0.05,{ }^{*} P<0.01\right)$. $\mathbf{b}$-j GUS histochemical staining in different tissues of transgenic rice. $\mathbf{b}$ young root; $\mathbf{c}$ young culm; $\mathbf{d}$ young leaf; e root; $\mathbf{f}$ culm; $\mathbf{g}$ leaf; $\mathbf{h}$ pistil; i stamen; j endosperm. Bars $=5 \mathrm{~mm}$. $\mathbf{k}$ Quantitative GUS assays of different tissues in transgenic rice

were immersed in staining buffer, that is $0.1 \mathrm{M}$ sodium phosphate buffer $(\mathrm{pH}=7.0)$ containing $1 \mathrm{mg} / \mathrm{ml} \mathrm{X-}$ Gluc, $0.5 \mathrm{mM} \mathrm{K} \mathrm{K}_{3}\left[\mathrm{Fe}(\mathrm{CN})_{6}\right], 0.5 \mathrm{mM} \quad \mathrm{K}_{4}\left[\mathrm{Fe}(\mathrm{CN})_{6}\right]$, $10 \mathrm{mM} \mathrm{Na}{ }_{2}$ EDTA, $0.1 \%(v / v)$ Triton X-100, and $10 \%$ (v/v) methanol, for $24 \mathrm{~h}$ at $37^{\circ} \mathrm{C}$ in the dark. Quantitative fluorometric GUS assays were performed by incubating the extracts with the 4-methyl-umbelliferyl- $\beta$-D-glucuronide (MUG) substrate in a lysis buffer for $15 \mathrm{~min}$ at $37^{\circ} \mathrm{C}$. GFP fluorescence was observed under a Leica M205C stereo microscope (Leica, Germany), and fluorescence was quantified using an EnSpire Multimode Plate Reader (PerkinElmer, USA) as described in a previous study $[30,38]$. The GFP fluorescence was excited at wavelength of $480 \mathrm{~nm}$ and measured at $520 \mathrm{~nm}$.

\section{RNA isolation and expression pattern analysis}

Total RNA was isolated using Plant RNA Kit according to the manufacturer's instructions (OMEGA Bio-tek, USA). For cDNA synthesis, we used the SuperQuickRT
MasterMix Kit (CWBIO, Beijing, China) with $2 \mu \mathrm{g}$ of total RNA as a template in a $20-\mu \mathrm{L}$ reaction mixture. Quantitative real-time PCR (qRT-PCR) was performed with an UltraSYBR Mixture Kit (CWBIO, Beijing, China) using the QuantStudio ${ }^{\mathrm{Tm}} 6$ Flex Real-Time PCR System (Life Technologies, USA). The relative expression level of each gene was calculated with normalizing to Actin1 (Accession NO. GQ339773) mRNA levels. Changes in expression were calculated using the $\Delta \Delta \mathrm{Ct}$ method. The gene-specific primers used are listed in Additional file 1: Table S1.

\section{Statistical analysis}

All data analyses were repeated three times with three replicate experiments independently. Standard deviations were indicated by error bars and the statistical significances were determined by one-way variance analysis. The mean differences were compared using Student's t test.
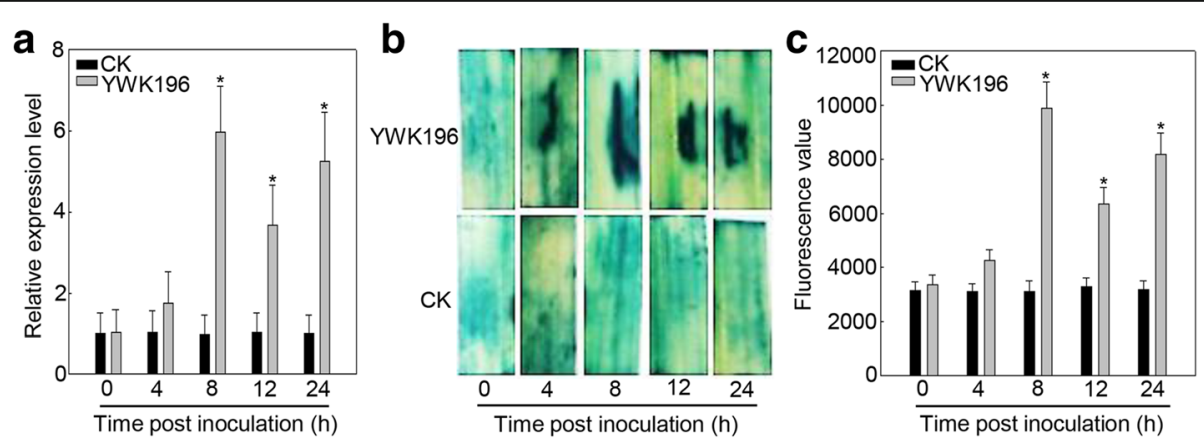

Fig. 2 Pathogen-inducible expression pattern of the maize gene GRMZM2G174449 and its promoter. a Time course of GRMZM2G1744449 expression after $R$. solani infection in 21-day-old seedlings. Asterisks indicate statistically significant differences, as determined by Student's t-tests $\left({ }^{*} P<0.05\right)$. b GUS histochemical staining in transgenic rice plants post inoculation with $R$. solani strain YWK196. c Quantitative GUS assays in transgenic rice leaves plants post inoculation with $R$. solani strain YWK196. Asterisks indicate statistically significant differences, as determined by Student's t-tests $(* 0<0.05)$ 


\section{Results}

\section{Characterization of $R$. solani-inducible gene} GRMZM2G174449

In our RNA-Seq data (Accession NO. SRP076058), GRMZM2G174449 was one of the up-regulated genes induced by $R$. solani strain YWK196 in B73. The expression patterns of tissue specific were further analyzed by qRT-PCR. As shown in Fig. 1a, GRMZM2G174449 was expressed at higher levels in young leaf, root, leaf, anther and endosperm tissues, but the gene exhibited low levels in other tissues of maize B73. A 6-fold increase of GRMZM2G174449 expression was observed $8 \mathrm{~h}$ post inoculation (hpi) with $R$. solani strain YWK196, with the expression levels maintained at 24 hpi (Fig. 2a), suggesting that the GRMZM2G174449 promoter could respond to $R$. solani in B73.

Bioinformatic analysis of the GRMZM2G174449 promoter To identify the regulatory mechanisms of the GRMZM2G174449 gene, we isolated its promoter region $(-1954$ to +46 , Accession NO. KY086278). According to the PLACE database [39], some putative cis-elements are predicted in $p G R M Z M 2 G 174449$ (Additional file 2: Figure S1). The TATA-box (5' -TATAAA-3') starts $76 \mathrm{bp}$ upstream of the ATG and $30 \mathrm{bp}$ upstream of the transcription start point (TSP), while a CAAT-box was located close to the TATA-box, which was 53 bp upstream of the TSP. In addition, the GRMZM2G174449 promoter also contains one pathogen- and salt-inducible element GT-1, GAAAAA [30, 40], five methyl jasmonate (MeJA)-responsive elements, CGTCA [41], two gibberellic acid (GA)-responsive elements, TAACAA [42], three abscisic acid (ABA)-responsive elements, ACGTG [43] and one auxin-responsive element, TGTCTC [44]. Eleven CANNTG elements which are known to be the binding site of the basic helix-loop-helix (bHLH) transcription factor [45], and one W-box (TTGACC) which is specifically recognized by the WRKY DNA binding proteins [46], were found in the promoter region.

The GRMZM2G174449 promoter derived expression pattern By using A. tumefaciens-mediated transformation, transgenic rice plants carrying the GRMZM2G174449 promoter-GUS construct were obtained. First, we examined the tissue-specific expression pattern of the GRMZM2G174449 promoter in transgenic rice. As shown in Fig. 1b-k, the GUS staining showed stronger enzymatic activity in the young leaf, root, leaf, anther and endosperm tissues than other tissues in the transgenic plants. Consistent with the GUS staining results, the GUS gene was expressed at high level in the young leaf, root, leaf, anther and endosperm tissues. Also, the rice result was similar to the tissue-specific expression pattern of GRMZM2G174449 gene in maize B73 (Fig. 1a).
We then examined the pathogen-inducible activity of the GRMZM2G174449 promoter by $R$. solani in transgenic rice leaves. After treatment with $R$. solani strain YWK196, the GUS activity was enhanced approximately 3 - fold by $8 \mathrm{hpi}$. It slowly declined by $24 \mathrm{hpi}$ but remained higher than the control (Fig. 2b-c). This induction pattern was similar to the expression pattern in maize too (Fig. 2a).

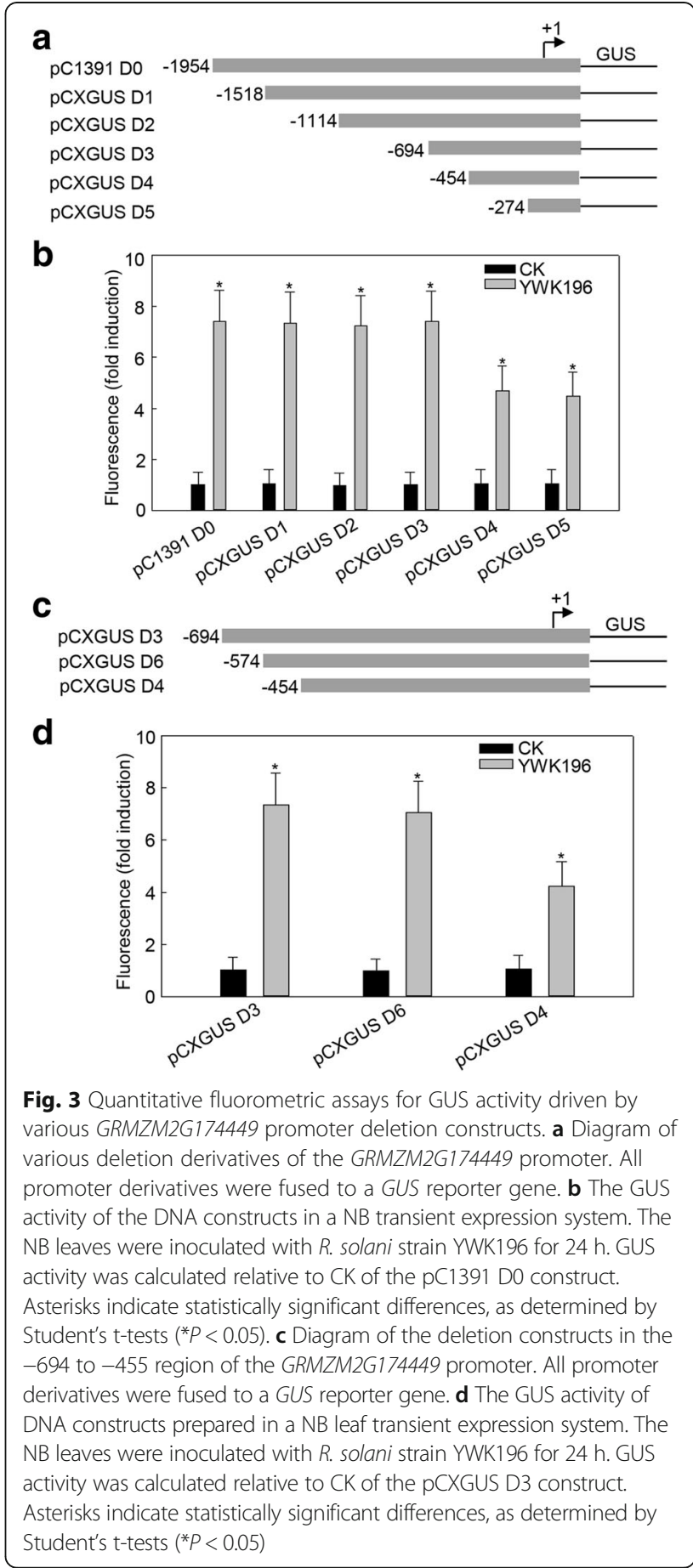


Deletion analysis of the GRMZM2G174449 promoter

To mine the specific regions of the GRMZM2G174449 promoter that are responsive to $R$. solani treatment, a series of $5^{\prime}$ deletions were made in the GRMZM2G174449 promoter region (Fig. 3a). Each construct was transiently expressed and induced by YWK196 inoculation in NB leaves, and GUS activity was assayed at $24 \mathrm{hpi}$. As shown in Fig. 3b, the full-length promoter (pC1391 D0) exhibited the highest level of inducible GUS activity, and equal induction levels were detected in constructs containing deletions up to -1518 (pCXGUS D1), -1114 (pCXGUS D2) and -694 (pCXGUS D3). However, this induction weakened in the construct containing deletion up to -454 (pCXGUS D4) and remained invariable in the pCXGUS D5 construct, but the induction levels in these two constructs were still higher than that in the control. These results indicated that the -694 to -455 and -274 to +46 fragments are two regions involved in the response to $R$. solani. Bioinformatic analysis of the two regions showed that the -694 to -455 region do not contain any known pathogen-inducible cis-elements, while the -274 to +46 region contains a cis-element GT-1 (GAAAAA) knowns as $R$. solani-inducible [30].

To identify the novel $R$. solani-inducible cis-elements in the -694 to -455 region, a $5^{\prime}$ deletion was further
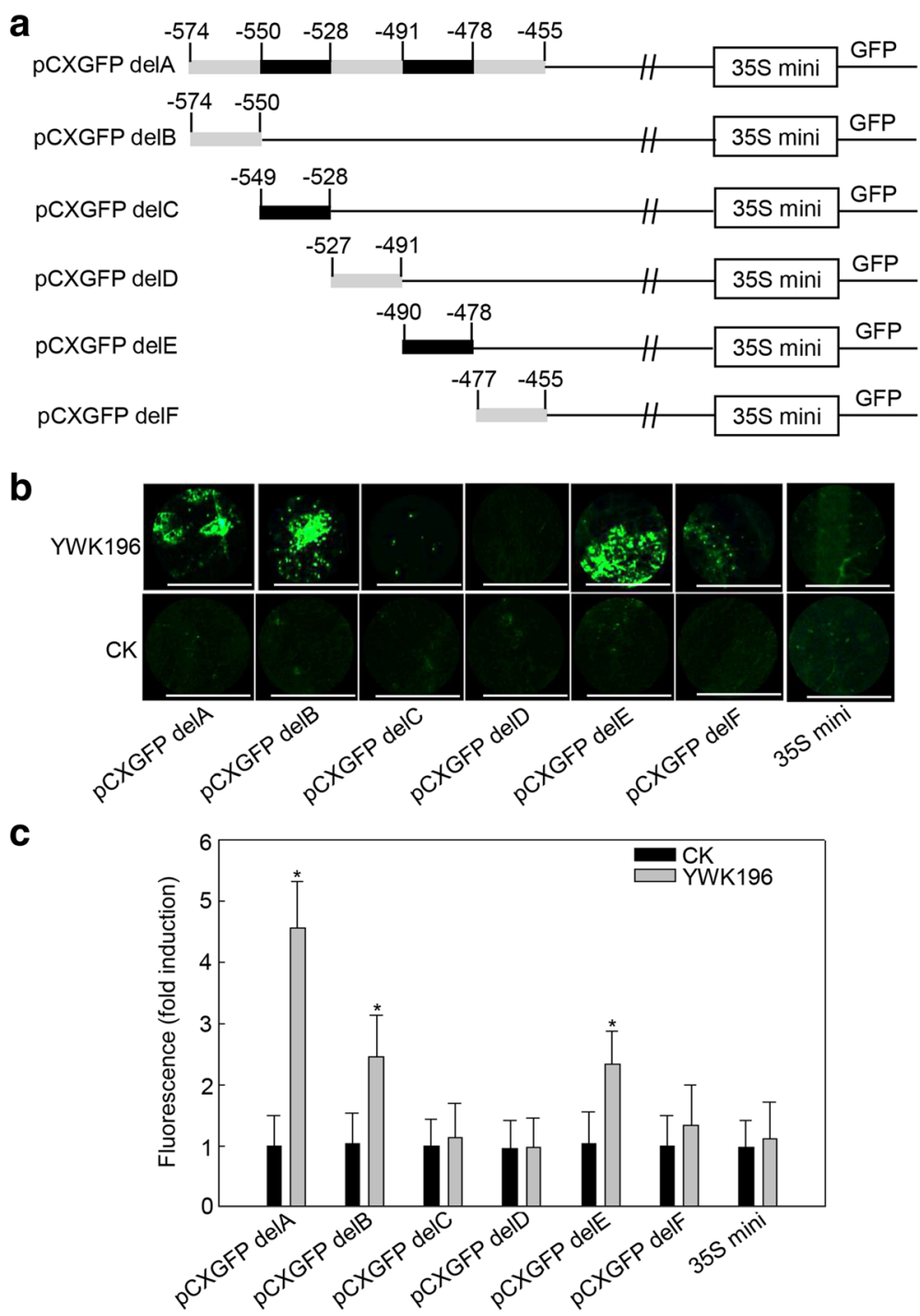

Fig. 4 The -574 to -550 and -490 to -478 fragments in the -574 to -455 region are responsive to $R$. solani. a Schematic diagram of the -574 to -455 region (pCXGFP delA) and the five deleted derivatives (pCXGFP delB to pCXGFP delF) used to express GFP in tobacco leaves. $\mathbf{b}$ GFP fluorescence assay of young and expanded symmetrical NB leaves infiltrated with pCXGFP delA or its derivatives after R. solani strain YWK196 infection for 24 h. Bars $=5 \mathrm{~mm}$. c Quantitative fluorometric assay of NB leaves. The fluorescence value was calculated relative to CK of pCXGFP delA. The $35 \mathrm{~S}$ minimum promoter was used as the negative control. Asterisks indicate statistically significant differences, as determined by Student's t-tests ( $P<0.05$ ) 
constructed in this region (Fig. 3c). These constructs were then tested in transient expression assays in NB leaves inoculated with YWK196 at 24 hpi. The construct with a deletion up to -574 (pCXGUS D6) showed almost equal GUS induction to that of the pCXGUS D3 construct. The pCXGUS D4 construct showed a roughly one-third reduction in GUS activity compared with that of the pCXGUS D3 and pCXGUS D6 constructs (Fig. 3d). These results indicated that the novel $R$. solani-inducible cis-elements are narrowly localized in the -574 to -455 region.

Two independent DNA fragments in the -574 to -455 region are responsive to $R$. solani

To narrow down the $R$. solani-inducible region of the -574 to -455 fragment, this region was further divided into five fragments of 10 to $30 \mathrm{bp}$ in length, which were individually fused with the $35 \mathrm{~S}$ minimum promoter (Fig. 4a). These constructs were then investigated in transient expression assays in NB leaves inoculated with YWK196 at 24 hpi (Fig. 4b-c). The pCXGFP delA construct showed GFP induction approximately 4.5-fold after treatment with YWK196, while the pCXGFP delB and pCXGFP delE constructs showed approximately 2.5-fold. In contrast, the pCXGFP delC, pCXGFP delD and pCXGFP delF constructs exhibited a very faint fluorescence signal. These results suggested that the 25-bp (GTACCTTGCTGATGGGCTCGGGGTG) and 13-bp (ACTACTATATCAA) sequences in the -574 to -455 region are responsive to $R$. solani.

\section{Characterization of the GCTGA and the TATAT are two $R$. solani-inducible cis-elements}

In our parallel study, two novel $R$. solani-inducible ciselements GTTGA and TATTT were identified in the other maize gene promoter, the GRMZM2G315431 promoter [34]. Interestingly, homologous sequences of GCTGA and TATAT were found in the 25-bp (GTACCTTGCTGATGGGCTCGGGGTG) and the 13bp (ACTACTATATCAA) DNA fragments, respectively. To identify whether the two sequences are core elements that involved in the response to $R$. solani, we produced

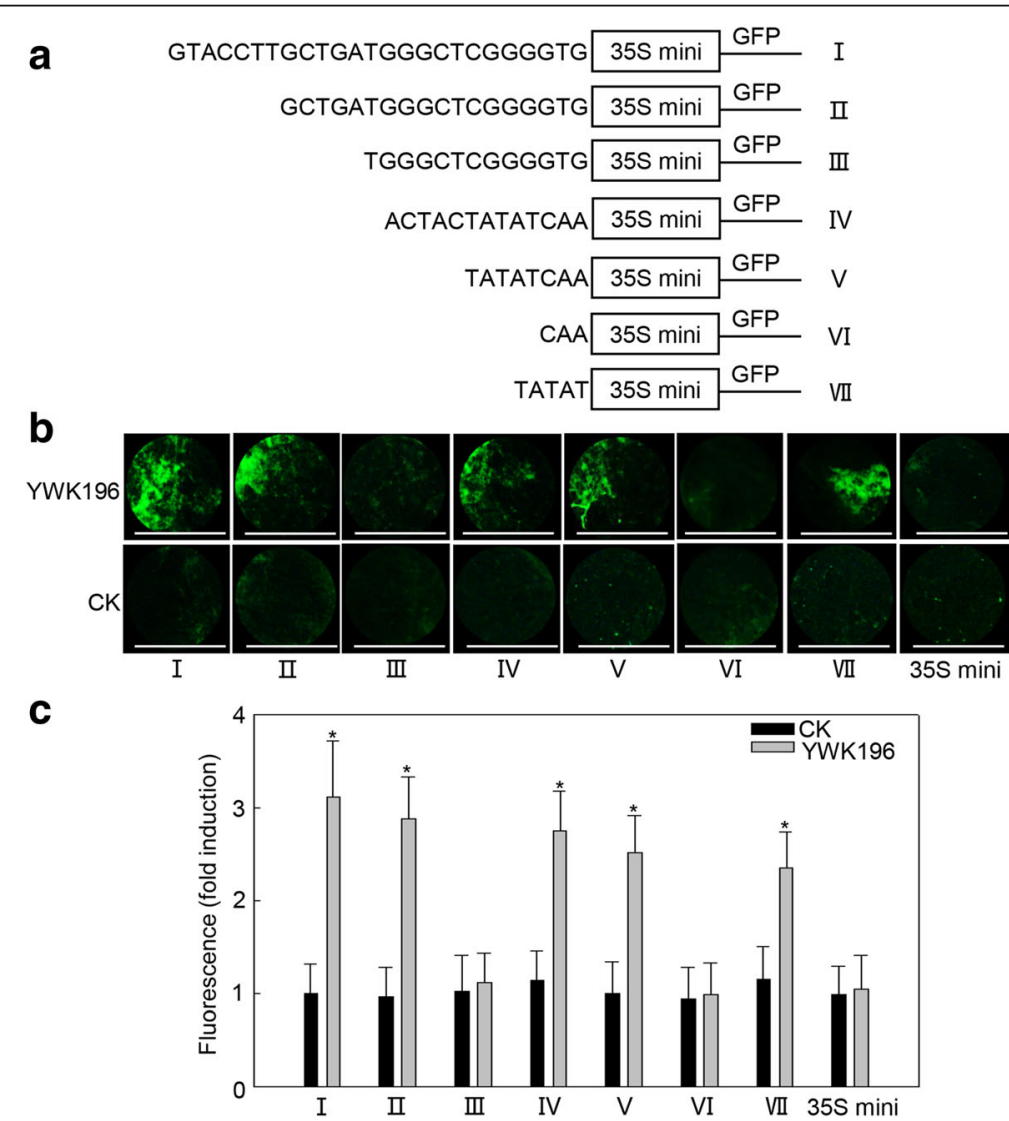

Fig. 5 The GCTGA and TATAT are two R. solani-inducible cis-elements. a Schematic diagram of the -574 to -550 (I), -490 to -478 (IV) and corresponding deleted derivatives (II, III, V, VI, VII) used to express GFP in NB leaves. b GFP fluorescence assay of young and expanded symmetrical NB leaves infiltrated with the six constructs after $R$. solani strain YWK196 infection for $24 \mathrm{~h}$. Bars $=5 \mathrm{~mm}$. c Quantitative fluorometric assay of NB leaves. Fluorescence value was calculated relative to CK of I. The 355 minimum promoter was used as the negative control. Asterisks indicate statistically significant differences, as determined by Student's t-tests $(* P<0.05)$ 
other deletion derivatives fused with the $35 \mathrm{~S}$ minimum promoter (Fig. 5a). The transient expression assays showed that deletion of GTACCTT in the 25-bp sequence exhibited an induction level (approximately 2.9 -fold) nearly equal to that of the 25-bp sequence. However, the GFP induction was completely lost after deleting the GCTGA sequence (Fig. 5b-c). Examination on the 13-bp sequence showed that deletion of the ACTAC did not affect the GFP induction, with the remaining TATAT sequence maintaining an equal level after deletion of the CAA at the $3^{\prime}$ end. Also, deletion of TATAT resulted in no GFP induction (Fig. 5b-c). These results indicated that GCTGA and TATAT are necessary for the $R$. solani induction of the 25-bp and 13-bp sequences, respectively.

To further determine whether these two cis-elements could respond to $R$. solani independently, we produced two constructs in which two tandem repeats of the two cis-elements $(2 \times$ GCTGA and $2 \times$ TATAT $)$ were fused to the $35 \mathrm{~S}$ minimum promoter. The full-length GRMZM2G174449 promoter and empty vector were used as controls. As shown in Fig. 6, the induction level of $2 \times$ GCTGA (approximately 3.9-fold) was almost equal to that of the full-length GRMZM2G174449 promoter (approximately 4.3-fold), and $R$. solani induced $2 \times$ TATAT to a level approximately two-thirds that of the GRMZM2G174449 promoter in the transient system. These data indicated that GCTGA and TATAT are two $R$. solani-inducible cis-elements.

\section{Functional validation of the GCTGA and TATAT} cis-elements in transgenic rice plants

To further confirm the results of the aforementioned transient assays, we generated GCTGA and TATAT transgenic rice plants using the constructs produced in Fig. 6 . Three $\mathrm{T}_{1}$ lines of each element were inoculated with

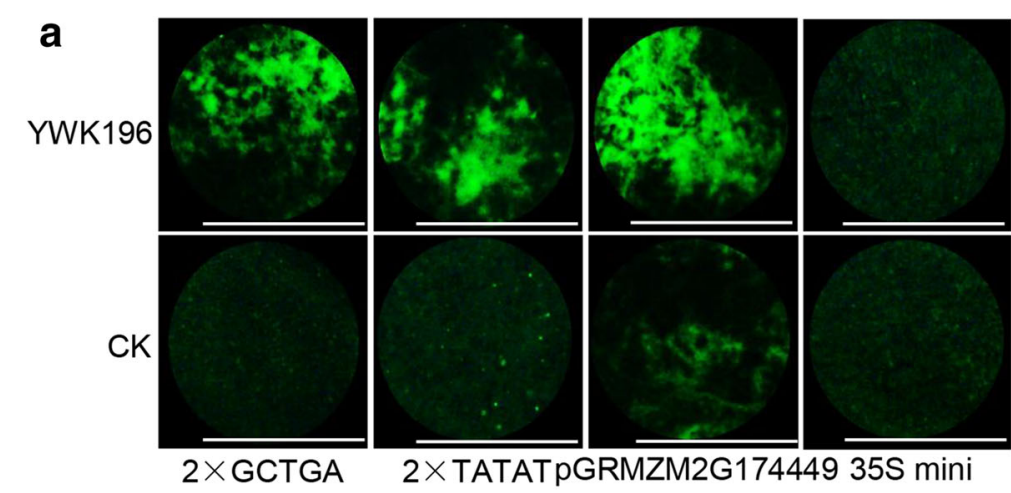

b

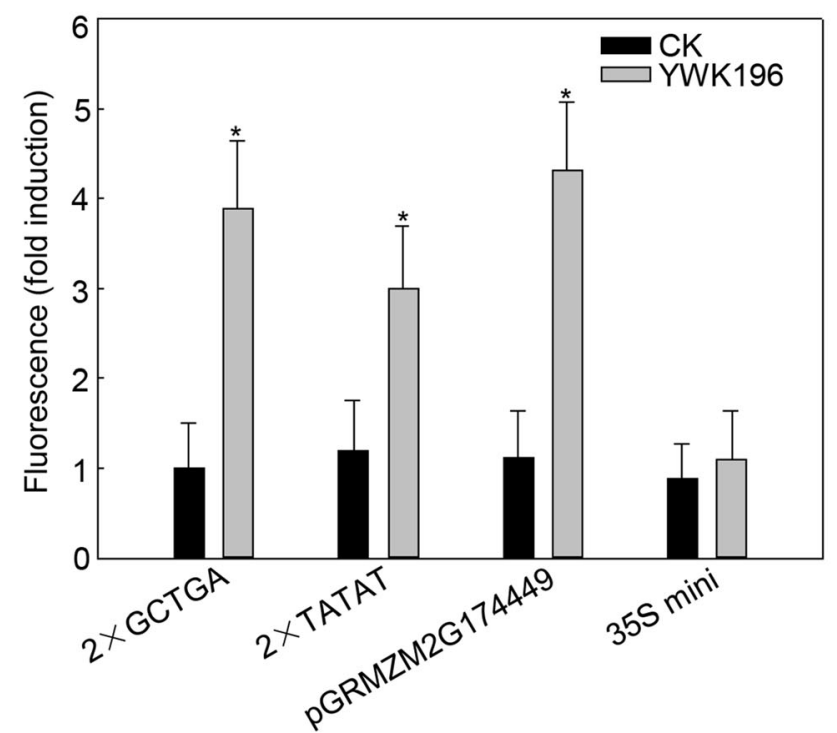

Fig. 6 Determination of GCTGA and TATAT induction by $R$. solani in NB leaves. a GFP fluorescence assay of NB leaves expressing $2 \times$ GCTGA and $2 \times$ TATAT after treatment with $R$. solani strain YWK196 for $24 \mathrm{~h}$. Bars $=5 \mathrm{~mm}$. b Quantitative fluorometric assay of NB leaves. The fluorescence value was calculated relative to CK of $2 \times$ GCTGA. The full-length GRMZM2G174449 promoter and 35S minimum promoter were used as the positive and negative controls, respectively. Asterisks indicate statistically significant differences, as determined by Student's t-tests $\left({ }^{*} P<0.05\right)$ 
YWK196 for 24 h. Leaves covered with PDA medium were used as native controls. As shown in Fig. 7, strong GFP fluorescence was observed in the $R$. solani-inoculated leaves of GCTGA and TATAT transgenic rice plants, and no clear GFP fluorescence was observed in the mock leaves and native control. These results were consistent with those in the NB transient-expression assays.

To investigate whether the two cis-elements were responsive to other $R$. solani strains and other rice pathogens, both transgenic lines used above were inoculated with $R$. solani strains LD16 and LD21, M. grisea strain RB21, Xoo strain PXO99 and Xoc strain RS105 for $24 \mathrm{~h}$. Leaves treated with media and water were used as controls. The GFP fluorescence was detected only in LD16- and LD21-inoculated leaves, while no considerable GFP was detected in leaves infected by other pathogens (Additional file 3: Figure S2). These results indicate that the two cis-elements are not responsible for the induction by M. grisea, Xoo or Xoc. Therefore, these two cis-elements might be specifically responded for $R$. solani induction.
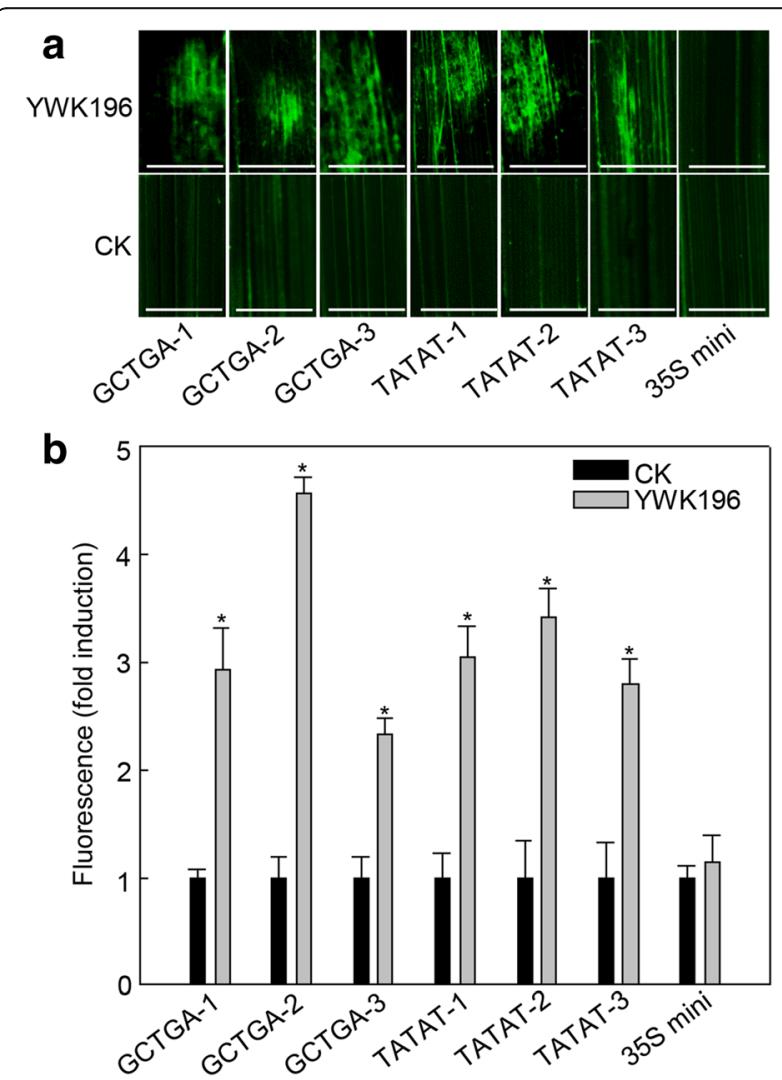

Fig. 7 Determination of GCTGA and TATAT induction by $R$. solani in the transgenic rice leaves. a GFP fluorescence assay of transgenic rice leaves inoculated with $R$. solani strain YWK196. Three $T_{1}$ lines of each element were used. Bars $=5 \mathrm{~mm}$. $\mathbf{b}$ Quantitative fluorometric assay of transgenic rice leaves post inoculation with $R$. solani. The fluorescence value was calculated relative to CK of GCTGA-1. The 355 minimum promoter was used as control. Asterisks indicate statistically significant differences, as determined by Student's t-tests $\left({ }^{*} P<0.05\right)$
Determination of the key bases of the GCTGA and TATAT elements

In order to identify the key bases of the two cis-elements, a series of point mutations were made and were fused to the $35 \mathrm{~S}$ minimum promoter. Compared with the fusion of GCTGA element, the construct fused with the sequence of CCTGA, TCTGA or GCTGG activated the similar level of GFP fluorescence post inoculation with $R$. solani. The constructs fused with GTTGA, GCAGA or GCTGT showed enhanced GFP fluorescence (1.21-, 1.14- and 1.18-fold, respectively), while the rest mutated constructs showed significantly attenuated GFP fluorescence in transient expression assay (Fig. 8a-b). Among the GFP attenuated subgroup, the constructs fused with ACTGA, GATGA or GCTGC were most closely to the GCTGA which regard to activate the GFP expression (Fig. 8a-b). Integrating with the results above, we could summarize a conserved motif NHWGN $(N=\mathrm{A} / \mathrm{T} / \mathrm{C} / \mathrm{G}, \mathrm{H}=\mathrm{A} / \mathrm{T} / \mathrm{C}, \mathrm{W}=\mathrm{A} / \mathrm{T})$ for the GCTGA cis-element (Fig. 8c). Compared with the construct carrying with TATAT, no mutated constructs could enhance the GFP fluorescence and only the construct of TACAT could activate the similar level of GFP fluorescence in transient expression assay. All the other mutated constructs showed significantly lessened GFP fluorescence in NB leaves (Fig. 8d-e). Among them, four mutated constructs were regarded to activate the GFP expression, including with the AATAT, the GATAT, the TTTAT and the TATTT, that is significantly higher than $35 \mathrm{~S}$ mini and very close to the construct fused with TATAT for the GFP fluorescence quantification (Fig. 8d, e). So we could summarize a conserved motif DWYWT $(\mathrm{D}=\mathrm{A} / \mathrm{T} / \mathrm{G}, \mathrm{W}=\mathrm{A} / \mathrm{T}, \mathrm{Y}=$ $\mathrm{T} / \mathrm{C})$ for the TATAT cis-element (Fig. 8f).

\section{Discussion}

Maize is an important cereal crop and industrial raw material. With the popularization of straw returning and high-yield cultivation technique, BLSB happened seriously year by year. It has become one of the most devastated diseases on maize production. Lack of resistant resources limited the selection of BLSB resistant varieties by traditional breeding, and no major resistance genes has been identified by now. Therefore, mining $R$. solani-inducible genes and studying the regulation mechanisms of resistance could provide new insights for resistance breeding.

In this study, we isolated the promoter of a $R$. solaniinducible gene GRMZM2G174449. This promoter was highly induced after treatment with $R$. solani for $8 \mathrm{~h}$ (Fig. 2b, c), indicating that the GRMZM2G174449 promoter is a typical $R$. solani-inducible promoter. Currently, constitutive promoters, such as the CaMV35S and ubiquitin promoters, are frequently used in the 


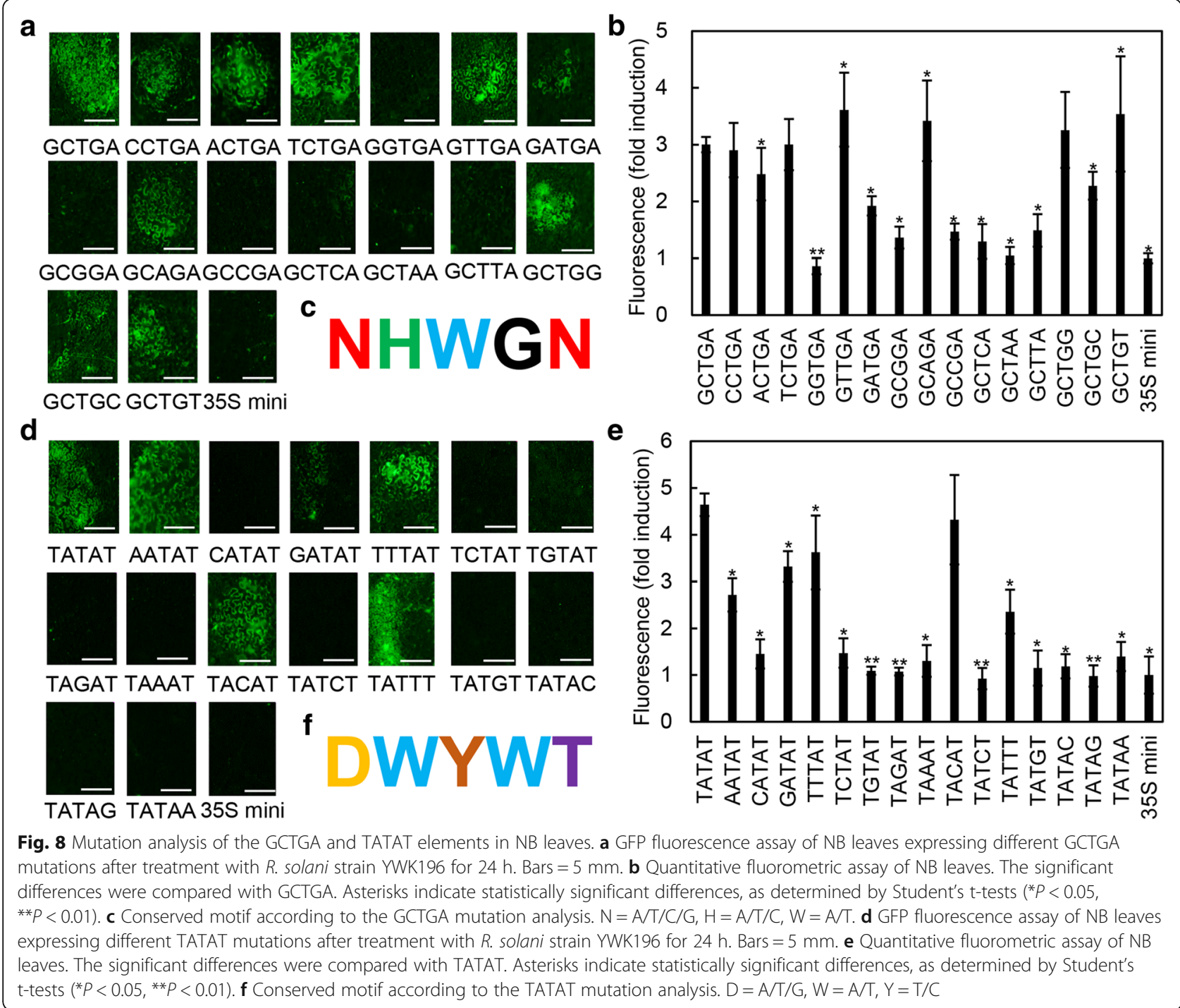

resistance improvement. However, constitutive expression of resistance genes is harmful to plant growth [2-4]. Pathogen-inducible promoters could regulate downstream genes quickly and accurately which have more benefits to breed the resistance crop than the constitutive promoters. Some $R$. solani-inducible promoters have been identified in maize and rice [30-34], and identification of the GRMZM2G174449 promoter further supplemented the BLSB resistant resources.

Deletion analysis showed that two regions ( -694 to -455 and -274 to +46 ) were involved in the response of GRMZM2G174449 promoter to $R$. solani (Fig. 3a, b). According to the prediction by PLACE, a GT-1 cis-element and a W-box were located in the -274 to +46 region (Additional file 2: Figure S1). The GT-1 element was first identified in the RBCS-3A promoter [47] and previous studies showed that it could be induced by Pseudomonas syringae and Phytophthora sojae [40, 48]. Recent study also showed that the GT-1 element played important roles in the rice defense-related gene Os2H16 responding to $R$. solani [30]. These results implied that there might be a conserved mechanism in maize and rice responding to $R$. solani, and the GT-1 signal is conserved in response to different pathogens and is one of the key factors in the basal immunity signal. The W-box is a specific binding site of WRKY transcription factors, and these transcription factors were reported to positively regulate the resistance to $R$. solani [49-51]. Overall, these results suggested that the GT-1 cis-element and W-box might play important roles in the -274 to +46 region.

Fine deletion analysis of the -694 to -455 region showed that the -574 to -550 and -490 to -478 were two important $R$. solani-inducible fragments (Fig. 4). In these two fragments, two elements, GCTGA and TATAT, were found to be homologous to another two 
identified $R$. solani-inducible cis-elements, GTTGA and TATTT, in the GRMZM2G315431 promoter [34]. Further experiments showed that the GCTGA and TATAT elements exhibited $R$. solani-inducible activity and did not respond to other tested pathogens (Fig. 6, Additional file 3: Figure S2). These results suggested that GTTGA and GCTGA, TATAT and TATTT could be defined as G (T/C) TGA and TAT (T/A) T, respectively. It indicated that the two genes might be regulated expression with the conserved transcription factors that mediated a signal pathway different from the basal immunity. However, the promoter of GRMZM2G174449 is different from the GRMZM2G315431 promoter. The derived GFP expression showed the relative lower expression in culm and delayer induction post $R$. solani inoculation than the GRMZM2G315431 promoter [34]. It could be partially explained that beside of two homologous cis-elements, there has only one additional pathogen-inducible element GT-1 in the promoter of GRMZM2G174449 (Additional file 2: Figure S1), and two GT-1 and one Wbox in the promoter of GRMZM2G315431 [34]. Mutation experiments showed that different mutations resulted different effects on the $R$. solani-inducible activities of the two cis-elements (Fig. 8a-b, d-e). According to these results, two conserved motifs NHWGN and DWYWT were summarized for the GCTGA and TATAT cis-elements, respectively (Fig. 8c-f). Moreover, we found that the inducible activity of GTTGA was higher than that of GCTGA, while the inducible activity of TATAT was higher than that of TATTT in the transient system (Fig. 8b-d). These results were consistent with that in the stable transgenic system (Additional file 4: Figure S3). Integrated with our recently reported two cases [30, 34], we believe that the transient system is presented good as the stable transgenic system in identifying the $R$. solani-inducible promoters. Future investigations into the interactions between the cis-elements and transcription factors will be meaningful, not only to improve our understanding of the molecular mechanisms of DNA-protein interactions during maize response to $R$. solani, but also for developing $R$. solani-resistant cultivars through breeding programs for maize and other crops.

\section{Conclusion}

Banded leaf and sheath blight, a highly destructive disease caused by the soil-born pathogen $R$. solani, leads to devastating reductions in maize yield. Although efforts have been made to produce resistant maize cultivars by traditional breeding and study the interactions between maize and $R$. solani, to date, banded leaf and sheath blight is not effectively controlled in most maize planting areas and the mechanisms are still not clear. Here, we isolated the GRMZM2G174449 promoter, and identified two DNA fragments, which could be highly induced by $R$. solani. We also mined the key cis-elements GCTGA and TATAT in the -574 to -550 and -490 to -478 fragments which involved in the maize response to $R$. solani. Finally, two conserved motifs NHWGN and DWYWT were summarized for the GCTGA and TATAT cis-elements, respectively. These findings not only enrich our knowledge of regulation of $R$. solani-inducible genes, but also provide new resources for resistance breeding.

\section{Additional files}

Additional file 1: Table S1. Information on the PCR primers used in this study. (XLSX $12 \mathrm{~kb}$ )

Additional file 2: Figure S1. Schematic map of the GRMZM2G174449 promoter with putative cis-elements. TSP, transcription start point; CANNTG-box, nematode-responsive box; ABA-box, ABA-responsive element; auxin-box, auxin-responsive element; GT-1-box, pathogen- and $\mathrm{NaCl}$-responsive element; W-box, elicitor-responsive element; GA-box, GA-responsive element; MeJA-box, MeJA-responsive element. (TIFF 84 kb)

Additional file 3: Figure S2. GFP expression driven by GCTGA and TATAT in the transgenic rice leaves post inoculation with $R$. solani strains LD16, LD21, M. grisea, Xoo and Xoc. a GFP fluorescence assay of transgenic rice leaves inoculated with $R$. solani strains LD16, LD21 and $M$. grisea. Three $T_{1}$ lines of each element were used. Bars $=5 \mathrm{~mm} . \mathrm{b}$ Quantitative fluorometric assay of transgenic rice leaves post inoculation with $R$. solani strains LD16, LD21 and M. grisea. The 35 S minimum promoter were used as the negative control. Asterisks indicate statistically significant differences, as determined by Student's t-tests $\left({ }^{*} P<0.05\right)$. $c$ Three $T_{1}$ lines of each element were inoculated with Xoo strain PXO99. The $35 \mathrm{~S}$ minimum promoter was used as the negative control. $d$ Three $\mathrm{T}_{1}$ lines of each element were inoculated with Xoc strain RS105. The $35 \mathrm{~S}$ minimum promoter was used as the negative control. Asterisks indicate statistically significant differences, as determined by Student's t-tests ( $\left.{ }^{*} P<0.05\right)$. (TIFF $2285 \mathrm{~kb}$ )

Additional file 4: Figure S3. Comparison of $R$. solani-inducible activities in the transgenic rice plants. a Comparison of $R$. solani-inducible activities between GCTGA and GTTGA in the transgenic rice plants. The $35 \mathrm{~S}$ minimum promoter was used as the negative control. The significant differences were compared with GTTGA. Asterisks indicate statistically significant differences, as determined by Student's t-tests ( $\left.{ }^{*} P<0.05\right)$. b Comparison of $R$. solani-inducible activities between TATAT and TATTT in the transgenic rice plants. The $35 \mathrm{~S}$ minimum promoter was used as the negative control. The significant differences were compared with TATAT. Asterisks indicate statistically significant differences, as determined by Student's t-tests ( $\left.{ }^{*} P<0.05\right)$. (TIFF $83 \mathrm{~kb}$ )

\section{Abbreviations}

ABA: Abscisic acid; BHLH: Basic helix-loop-helix; BLSB: Banded leaf and sheath blight; CaMV35S: Cauliflower mosaic virus 35S; GA: Gibberellic acid; GUS: $\beta$ glucuronidase; hpi: Hours post inoculation; MeJA: Methyl jasmonate; MUG: 4methyl-umbelliferyl-ß-D-glucuronide; NB: Nicotiana benthamiana; qRTPCR: Quantitative real-time PCR; RNA-seq: RNA sequencing; TSP: Transcription start point; Xoc: Xanthomonas oryzae pv. oryzicola; Xoo: Xanthomonas oryzae pv. oryzae

\section{Acknowledgements}

Not applicable

\section{Funding}

This work was supported by the National Natural Science Foundation of China $(31,601,279,31,771,748)$, the Shandong Modern Agricultural Technology \& Industry system (SDAIT-17-06), the Key Research and 
Development Program of Shandong Province (2017GNC10104) and the Funds of Shandong "Double Tops" Program (2017).

\section{Availability of data and materials}

The datasets supporting the conclusions of this article are included within the article and its additional files. All plant materials and pathogens were obtained from Shandong Agricultural University, Taian, China.

\section{Authors' contributions}

$Z C$ and $X D$ conceived and designed the experiments. FY, NL, JC and YS performed the experiments. FY, JC, LK and XD analyzed the data. ZC and NL wrote the manuscript. All authors read and approved the final manuscript.

\section{Ethics approval and consent to participate}

Not applicable

\section{Consent for publication}

Not applicable.

\section{Competing interests}

The authors declare that they have no competing interests.

\section{Publisher's Note}

Springer Nature remains neutral with regard to jurisdictional claims in published maps and institutional affiliations.

\section{Author details}

'State Key Laboratory of Crop Biology, College of Agronomy, Shandong Agricultural University, Tai an 271018, Shandong Province, People's Republic of China. ${ }^{2}$ Shandong Provincial Key Laboratory of Agricultural Microbiology, College of Plant Protection, Shandong Agricultural University, Tai an 271018, Shandong Province, People's Republic of China.

\section{Received: 17 September 2017 Accepted: 22 November 2017} Published online: 04 December 2017

\section{References}

1. Herrera-Estrella $L$, Simpson J. Genetically engineered resistance to bacterial and fungal pathogens. World J Microb Biot. 1995;11(4):383-92.

2. Zhang J, Peng Y, Guo Z. Constitutive expression of pathogen-inducible OSWRKY31 enhances disease resistance and affects root growth and auxin response in transgenic rice plants. Cell Res. 2008;18(4):508-21.

3. Flachowsky H, Szankowski I, Fischer TC, Richter K, Peil A, Höfer M, et al. Transgenic apple plants overexpressing the $L c$ gene of maize show an altered growth habit and increased resistance to apple scab and fire blight. Planta. 2010;231(3):623-35.

4. Nadal A, Montero M, Company N, Badosa E, Messeguer J, Montesinos L, et al. Constitutive expression of transgenes encoding derivatives of the synthetic antimicrobial peptide BP100: impact on rice host plant fitness. BMC Plant Biol. 2012;12(1):159-79.

5. Gurr SJ, Rushton PJ. Engineering plants with increased disease resistance: how are we going to express it? Trends Biotechnol. 2005;23(6):275-82.

6. Chai C, Lin Y, Shen D, Wu Y, Li H, Dou D. Identification and functional characterization of the soybean GmaPPO12 promoter conferring Phytophthora sojae induced expression. PLoS One. 2013;8(6):e67670.

7. Zou X, Song E, Peng A, He Y, Xu L, Lei T, et al. Activation of three pathogen-inducible promoters in transgenic citrus (Citrus sinensis Osbeck) after Xanthomonas axonopodis pv. citri infection and wounding. Plant Cell Tissue Organ Cult. 2014;117(1):85-98.

8. Tao Y, Wang F, Jia D, Li J, Zhang Y, Jia C, et al. Cloning and functional analysis of the promoter of a stress-inducible gene ( $\mathrm{m} m \mathrm{RXO1}$ ) in maize. Plant Mol Biol Rep. 2015;33(2):200-8.

9. Rushton PJ, Reinstädler A, Lipka V, Lippok B, Somssich IE. Synthetic plant promoters containing defined regulatory elements provide novel insights into pathogen- and wound-induced signaling. Plant Cell. 2002;14(4):749-62.

10. Kobayashi M, Yoshioka M, Asai S, Nomura H, Kuchimura K, Mori H, et al. StCDPK5 confers resistance to late blight pathogen but increases susceptibility to early blight pathogen in potato via reactive oxygen species burst. New Phytol. 2012;196(1):223-37.

11. Helliwell EE, Wang Q, Yang Y. Transgenic rice with inducible ethylene production exhibits broad-spectrum disease resistance to the fungal pathogens Magnaporthe oryzae and Rhizoctonia solani. Plant Biotechnol J. 2013;11(1):33-42

12. Van der Does D, Leon-Reyes A, Koornneef A, Van Verk MC, Rodenburg N, Pauwels $L$, et al. Salicylic acid suppresses jasmonic acid signaling downstream of SCF ${ }^{\text {COl1 }}$-JAZ by targeting GCC promoter motifs via transcription factor ORA59. Plant Cell. 2013;25(2):744-61.

13. Huang P, Catinot J, Zimmerli L. Ethylene response factors in Arabidopsis immunity. J Exp Bot. 2015;67(2):1231-41.

14. Laloi C, Mestres-Ortega D, Marco Y, Meyer Y, Reichheld JP. The Arabidopsis cytosolic thioredoxin $h 5$ gene induction by oxidative stress and its $\mathrm{W}$-boxmediated response to pathogen elicitor. Plant Physiol. 2004;134(3):1006-16.

15. Yamamoto S, Nakano T, Suzuki K, Shinshi H. Elicitor-induced activation of transcription via $\mathrm{W}$ box-related cis-acting elements from a basic chitinase gene by WRKY transcription factors in tobacco. Biochim Biophys Acta. 2004; 1679(3):279-87.

16. Gao $Y$, Zan $X L$, Wu XF, Yao L, Chen $Y L$, Jia SW, et al. Identification of fungus responsive cis-acting element in the promoter of Brassica juncea chitinase gene, BjCHI1. Plant Sci. 2014;215:190-8.

17. Kirsch C, Takamiya-Wik M, Schmelzer E, Hahlbrock K, Somssich IE. A novel regulatory element involved in rapid activation of parsley ELI7 gene family members by fungal elicitor or pathogen infection. Mol Plant Pathol. 2000; 1(4):243-51.

18. Alves MS, Dadalto SP, Gonçalves AB, De Souza GB, Barros VA, Fietto LG. Plant bZIP transcription factors responsive to pathogens: a review. Int J Mol Sci. 2013;14(4):7815-28.

19. Miyamoto K, Shimizu T, Lin F, Sainsbury F, Thuenemann E, Lomonossoff G, et al. Identification of an E-box motif responsible for the expression of jasmonic acid-induced chitinase gene OsChia4a in rice. J Plant Physiol. 2012 169(6):621-7.

20. Cai M, Qiu D, Yuan T, Ding X, Li H, Duan L, et al. Identification of novel pathogen-responsive cis-elements and their binding proteins in the promoter of OsWRKY13, a gene regulating rice disease resistance. Plant Cell Environ. 2008;31(1):86-96.

21. Yeri SB, Bhat RS, Kuruvinashetti MS. Functional analysis of synthetic promoters containing pathogen-responsive cis-elements. Mol Plant Breed. 2013:4(34):270-6.

22. González-Vera AD, Bernardes-de-Assis J, Zala M, McDonald BA, CorreaVictoria F, Graterol-Matute EJ, et al. Divergence between sympatric rice- and maize-infecting populations of Rhizoctonia solani AG-1 IA from Latin America. Phytopathology. 2010;100(2):172-82.

23. Yellaredddygari SKR, Reddy MS, Kloepper JW, Lawrence KS, Fadamiro H. Rice sheath blight: a review of disease and pathogen management approaches. I Plant Pathol Microbiol. 2014;5:4

24. Wang $Y$, Pinson SRM, Fjellstrom RG, Tabien RE. Phenotypic gain from introgression of two QTL, QSB9-2 and qSB12-1, for rice sheath blight resistance. Mol Breed. 2012;30(1):293-303.

25. Zuo S, Yin Y, Pan C, Chen Z, Zhang Y, Gu S, et al. Fine mapping of $a S B-11^{L E}$, the QTL that confers partial resistance to rice sheath blight. Theor Appl Genet. 2013;126(5):1257-72.

26. Li N, Kong L, Zhou W, Zhang X, Wei S, Ding X, et al. Overexpression of Os2H16 enhances resistance to phytopathogens and tolerance to drought stress in rice. Plant Cell Tissue Organ Cult. 2013;115(3):429-41.

27. Molla KA, Karmakar S, Chanda PK, Ghosh S, Sarkar SN, Datta SK, et al. Rice oxalate oxidase gene driven by green tissue-specific promoter increases tolerance to sheath blight pathogen (Rhizoctonia solani) in transgenic rice. Mol Plant Pathol. 2013;14(9):910-22.

28. Pan X, Li Y, Zhang H, Huang R, Liu W, Ming J, et al. Expression of signaling and defense-related genes mediated by over-expression of JERF1, and increased resistance to sheath blight in rice. Plant Pathol. 2014;63(1):109-16.

29. Richa K, Tiwari IM, Devanna BN, Botella JR, Sharma V, Sharma TR, et al. Nove chitinase gene. LOC_Os11g47510 from indica rice tetep provides enhanced resistance against sheath blight pathogen Rhizoctonia solani in rice. Front Plant Sci. 2017. doi:10.3389/fpls.2017.00596.

30. Li N, Wei ST, Chen J, Yang FF, Kong LG, Chen CX, et al. OsASR2 regulates the expression of a defense-related gene, Os2H16, by targeting the GT-1 cis-element. Plant Biotechnol J. 2017; https://doi.org/10.1111/pbi.12827.

31. Roby D, Broglie K, Cressman R, Biddle P, Chet IL, Broglie R. Activation of a bean chitinase promoter in transgenic tobacco plants by phytopathogenic fungi. Plant Cell. 1990;2(10):999-1007.

32. Samac DA Shah DM. Developmental and pathogen-induced activation of the Arabidopsis acidic chitinase promoter. Plant Cell. 1991;3(10):1063-72. 
33. Perl-Treves R, Foley RC, Chen W, Singh KB. Early induction of the Arabidopsis GSTF8 promoter by specific strains of the fungal pathogen Rhizoctonia solani. Mol Plant-Microbe Interact. 2004;17(1):70-80.

34. Li N, Chen J, Yang F, Wei S, Kong L, Ding X, et al. Identification of two novel Rhizoctonia solani-inducible cis-acting elements in the promoter of the maize gene, GRMZM2G315431. Sci Rep. 2017;7:42059.

35. Chen S, Songkumarn P, Liu J, Wang G. A versatile zero background T-vector system for gene cloning and functional genomics. Plant Physiol. 2009; 150(3):1111-21.

36. Lin Y, Chen H, Cao Y, Wu C, Wen J, Li Y, et al. Establishment of highefficiency Agrobacterium-mediated genetic transformation system of Mudanjiang 8. Acta Agron Sin. 2002;28(3):294-300

37. Lee SH, Kim JC, Lee MS, Heo WD, Seo HY, Yoon HW, et al. Identification of a novel divergent calmodulin isoform from soybean which has differential ability to activate calmodulin-dependent enzymes. J Biol Chem. 1995; 270(37):21806-12.

38. McLellan H, Boevink PC, Armstrong MR, Pritchard L, Gomez S, Moraleset J, et al. An RxLR effector from Phytophthora infestans prevents re-localization of two plant NAC transcription factors from the endoplasmic reticulum to the nucleus. PLoS Pathog. 2013;9(10):e1003670.

39. Higo $\mathrm{K}$, Ugawa $\mathrm{Y}$, Iwamoto M, Korenaga T. Plant cis-acting regulatory DNA elements (PLACE) database. Nucl Acids Res. 1999;27(1):297-300.

40. Park HC, Kim ML, Kang YH, Jeon JM, Yoo JH, Kim MC, et al. Pathogen- and $\mathrm{NaCl}-$ induced expression of the SCaM-4 promoter is mediated in part by a GT-1 box that interacts with a GT-1-like transcription factor. Plant Physiol. 2004:135(4):2150-61

41. Wang Y, Liu G, Yan X, Wei Z, Xu Z. MeJA-inducible expression of the heterologous JAZ2 promoter from Arabidopsis in Populus trichocarpa protoplasts. J Plant Dis Protect. 2011;118(2):69-74.

42. Woodger FJ, Millar A, Murray F, Jacobsen JV, Gubler F. The role of GAMYB transcription factors in GA-regulated gene expression. J Plant Growth Regul. 2003;22(2):176-84.

43. Li J, Sima W, Ouyang B, Luo Z, Yang C, Ye Z, et al. Identification and expression pattern of a ZPR1 gene in wild tomato (Solanum Pennellii). Plant Mol Biol Rep. 2013:31(2):409-17.

44. Li Y, Liu BZ, Shi X, Hagen G, Guilfoyle TJ. An auxin-inducible element in soybean SAUR promoters. Plant Physiol. 1994:106(1):37-43.

45. Wang J, Hu J, Qian Q, Xue H. LC2 and OsVIL2 promote rice flowering by photoperiod-induced epigenetic silencing of OsLF. Mol Plant. 2013:6(2):514-27.

46. Li C, He X, Luo X, Xu L, Liu L, Min L, et al. Cotton WRKY1 mediates the plant defense-to-development transition during infection of cotton by Verticillium dahlia by activating JASMONATE ZIM-DOMAIN1 expression. Plant Physiol. 2014;166(4):2179-94.

47. Green PJ, Kay SA, Chua NH. Sequence-specific interactions of a pea nuclear factor with light-responsive elements upstream of the rbcS-3A gene. EMBO J. 1987;6(9):2543-9.

48. Chai CY, Lin YL, Wu YR, Shen DL, Dou DL. Identification and functional characterization of the Phytophthora sojae induced-promoter of the soybean GmDRRP gene. Acta Phytopathol Sin. 2015;45(1):57-66.

49. Wang H, Meng J, Peng X, Tang X, Zhou P, Xiang J, et al. Rice WRKY 4 acts as a transcriptional activator mediating defense responses toward Rhizoctonia solani, the causing agent of rice sheath blight. Plant Mol Biol. 2015;89(1-2):157-71.

50. Yan Y, Jia H, Wang F, Wang C, Liu S, Guo X. Overexpression of GhWRKY27a reduces tolerance to drought stress and resistance to Rhizoctonia solani infection in transgenic Nicotiana benthamiana. Front Physiol. 2015;6:265.

51. Peng $X$, Wang $H$, Jang JC, Xiao T, He H, Jiang D, et al. OsWRKY80-OsWRKY 4 module as a positive regulatory circuit in rice resistance against Rhizoctonia solani. Rice. 2016;9:63.

\section{Submit your next manuscript to BioMed Central and we will help you at every step:}

- We accept pre-submission inquiries

- Our selector tool helps you to find the most relevant journal

- We provide round the clock customer support

- Convenient online submission

- Thorough peer review

- Inclusion in PubMed and all major indexing services

- Maximum visibility for your research

Submit your manuscript at www.biomedcentral.com/submit

CBiomed Central 